\title{
Modified Moving Least Squares with Polynomial Bases for Scattered Data Approximation
}

\author{
Grand Roman Joldes*1, Habibullah Amin Chowdhury ${ }^{1}$, Adam Wittek ${ }^{1}$, Barry \\ Doyle $^{1}$ and Karol Miller ${ }^{1,2}$ \\ ${ }^{1}$ Intelligent Systems for Medicine Laboratory, School of Mechanical and Chemical Engineering, \\ The University of Western Australia, 35 Stirling Highway, Crawley/Perth WA 6009, AUSTRALIA \\ ${ }^{2}$ School of Engineering. Cardiff University, UK
}

\begin{abstract}
One common problem encountered in many fields is the generation of surfaces based on values at irregularly distributed nodes. To tackle such problems, we present a modified, robust Moving Least Squares (MLS) method for scattered data smoothing and approximation. The error functional used in the derivation of the classical MLS approximation is augmented with additional terms based on the coefficients of the polynomial base functions. This allows quadratic base functions to be used with the same size of the support domain as linear base functions, resulting in better approximation capability. The increased robustness of the modified MLS method to irregular nodal distributions makes it suitable for use across many fields. The analysis is supported by several univariate and bivariate examples.
\end{abstract}

KEYWORDS: Moving Least Squares; random point distribution; scattered data approximation; robust shape function generation

*Corresponding Author, E-mail: grand.joldes@,uwa.edu.au, Phone: +618 6488 3125, Fax: +618 64881024 


\section{INTRODUCTION}

Interpolation of scattered data is a common problem in many engineering fields. We are mostly interested in problems which occur in medicine, especially medical image registration. A reliable method for interpolating scattered data is required both for visualization as well as for performing numerical computations (using state of the art meshless methods [1-5]).

The use of the Moving Least Squares (MLS) method for smoothing and approximating scattered data was proposed by Lancaster and Salkauskas [6]. Since then, due to the smoothness and continuity of the approximation field it generates, the method has been adopted in multiple fields, such as surface definition from points [7], approximation of implicit surfaces [8], animations [9], simulations [2, 10], and computational biomechanics $[3,11]$.

In most applications a local evaluation of the approximating function is desired, and therefore the compact support domain for each data point (the domain over which the shape function associated with the point is non-zero) is chosen as a sphere or a parallelogram box centered on the point $[1,2,6]$. This simplifies the computation of influence domains for a given point, i.e. finding which support domains contain that point. Each data point has an associated dilatation parameter, which characterizes the size of its compact support domain.

Not all node distributions can be used in numerical computations, as shape functions cannot always be computed over the entire problem domain. A valid node distribution is referred to as an "admissible node distribution" [1]. The number of admissible node distributions can be increased by increasing the size of the support domains (the dilatation parameters), but this leads to an increased number of data points inside influence domains, an increased number of shape functions covering a local area, more linearly-dependent shape functions in the local area and increased computational cost. 
Higher order polynomial base functions can create better approximations for complex data distributions. Nevertheless, as the degree of the polynomial base function increases, it becomes more difficult to ensure the independence of the shape functions, and the least squares minimization problem becomes ill-posed. The usual solution requires increased size of the support domains so that more data points are included in the influence domain for each evaluation point.

There have been several techniques developed for handling ill-posed least squares problems. A first class of techniques, developed in the context of regression analysis in statistics, consists of regularization methods. These include the Tikhonov-Miller regularization [12-14], also known as ridge regression or constrained linear regression method, the least absolute shrinkage and selection operator (LASSO) [15], or a combination of the two, known as elastic net regularization [16]. These techniques have been developed for linear regression of statistical data, which is equivalent to a least squares optimization using linear basis. These techniques can be easily extended to weighted least squares regularization and higher degree polynomial basis functions. In the context of moving least squares (MLS) and our intended applications, only the Tikhonov-Miller regularization is of interest, as the other two techniques lead to nonsmooth approximations.

Several techniques have been proposed for handling a singular moment matrix in the context of MLS based mesh-free methods, such as perturbation of nodal positions, coordinate transformation, or the matrix triangularization algorithm (MTA) [2]. These techniques have been developed in the context of the point interpolation method (PIM), and therefore assume that sufficient nodes exist in the support domain; they also do not ensure the smoothness and continuity of the approximation.

This paper presents a modified MLS (MMLS) approximation which allows higher order polynomial base functions to be used under the same conditions as lower degree base functions. This is achieved by augmenting the error functional used in the derivation of the MLS shape functions with additional terms based on the coefficients of the polynomial base functions, therefore introducing additional constraints. The technique used is similar to the Tikhonov-Miller regularization. 
The paper is organized as follows: the classical MLS approximation, the modified MLS approximation with second order polynomial bases and important properties of the proposed approximation are discussed in the next Section, several univariate and bivariate examples are presented in Section 3, followed by discussion and conclusions in Section 4. 


\section{THEORY}

\subsection{The classical MLS approximation}

We succinctly present the classical MLS approximation with polynomial bases using the notations and derivation procedure from [2]. This will form the basis for the development of the modified MLS approximation in the next section. Given $n$ data points (nodes) located at positions $\mathbf{x}_{j}$ in $\mathbb{R}^{d}, j=1 . . n$, we can obtain a function $u^{h}(\mathbf{x})$ that approximates the given scalar values $u_{j}$ at points $\mathbf{x}_{j}$ by minimizing the error functional

$$
J(\mathbf{x})=\sum_{j=1}^{n}\left[\left(u^{h}\left(\mathbf{x}_{j}\right)-u_{j}\right)^{2} w\left(\left\|\mathbf{x}-\mathbf{x}_{j}\right\|\right)\right]
$$

where the error between the defined function and the given scalar values is weighted using the positive weight function $w$ based on the Euclidean distances between the evaluation point and the positions of the nodes. We use $\|$.$\| as the notation for Euclidean$ distance.

The function $u^{h}(\mathbf{x})$ is chosen as a polynomial

$$
u^{h}(\mathbf{x})=\sum_{i=1}^{m} p_{i}(\mathbf{x}) a_{i}(\mathbf{x})=\mathbf{p}^{T}(\mathbf{x}) \boldsymbol{a}(\mathbf{x})
$$

where $m$ is the number of terms in the bases $\mathbf{p}(\mathbf{x})$ and $a_{i}(\mathbf{x})$ are the coefficients that depend on spatial coordinates $\mathbf{x}$ (due to the weight functions which depend on $\mathbf{x}$ ). For example, commonly used bases and the corresponding coefficients in $2 \mathrm{D}$ are:

- linear bases:

$$
\mathbf{p}^{T}(\mathbf{x})=[1, x, y], \quad \mathbf{a}^{T}(\mathbf{x})=\left[a_{1}, a_{x}, a_{y}\right]
$$

- quadratic bases:

$$
\mathbf{p}^{T}(\mathbf{x})=\left[1, x, y, x^{2}, x y, y^{2}\right], \quad \mathbf{a}^{T}(\mathbf{x})=\left[a_{1}, a_{x}, a_{y}, a_{x^{2}}, a_{x y}, a_{y^{2}}\right]
$$

The coefficients $a_{i}(\mathbf{x})$ are obtained by minimizing the weighted least-square functional $J(\mathbf{x})$ given by (1), which can be rewritten in matrix form as:

$$
\mathbf{J}=(\mathbf{P a}-\mathbf{u})^{T} \mathbf{W}(\mathbf{P a}-\mathbf{u})
$$

where 


$$
\begin{gathered}
\mathbf{u}^{T}=\left[u_{1}, u_{2}, \ldots u_{n}\right] \\
\mathbf{P}=\left[\begin{array}{cccc}
p_{1}\left(\mathbf{x}_{1}\right) & p_{2}\left(\mathbf{x}_{1}\right) & \cdots & p_{m}\left(\mathbf{x}_{1}\right) \\
p_{1}\left(\mathbf{x}_{2}\right) & p_{2}\left(\mathbf{x}_{2}\right) & \cdots & p_{m}\left(\mathbf{x}_{2}\right) \\
\vdots & \vdots & \ddots & \vdots \\
p_{1}\left(\mathbf{x}_{n}\right) & p_{2}\left(\mathbf{x}_{n}\right) & \cdots & p_{m}\left(\mathbf{x}_{n}\right)
\end{array}\right]=\left[\begin{array}{c}
\mathbf{p}\left(\mathbf{x}_{1}\right)^{T} \\
\mathbf{p}\left(\mathbf{x}_{2}\right)^{T} \\
\vdots \\
\mathbf{p}\left(\mathbf{x}_{n}\right)^{T}
\end{array}\right] \\
\mathbf{W}=\left[\begin{array}{cccc}
w\left(\left\|\mathbf{x}-\mathbf{x}_{1}\right\|\right) & 0 & \cdots & 0 \\
0 & w\left(\left\|\mathbf{x}-\mathbf{x}_{2}\right\|\right) & \cdots & 0 \\
\vdots & \vdots & \ddots & \vdots \\
0 & 0 & \cdots & w\left(\left\|\mathbf{x}-\mathbf{x}_{n}\right\|\right)
\end{array}\right]
\end{gathered}
$$

We can minimize (5) by setting the partial derivatives of the error functional $\mathbf{J}$ to zero:

$$
\frac{\partial \mathbf{J}}{\partial \mathbf{a}}=\mathbf{P}^{T} \mathbf{W P a}(\mathbf{x})-\mathbf{P}^{T} \mathbf{W u}=0
$$

If the square matrix

$$
\mathbf{M}=\mathbf{P}^{T} \mathbf{W P}
$$

also known as the moment matrix, is non-singular, the values of the coefficients at the evaluation point are obtained as:

$$
\mathbf{a}(\mathbf{x})=\left(\mathbf{P}^{T} \mathbf{W P}\right)^{-1} \mathbf{P}^{T} \mathbf{W u}
$$

The approximation function can therefore be expressed, based on (2), as:

$$
u^{h}(\boldsymbol{x})=\mathbf{P}^{T}\left(\mathbf{P}^{T} \mathbf{W} \mathbf{P}\right)^{-1} \mathbf{P}^{T} \mathbf{W u}=\sum_{j=1}^{n} \emptyset_{j}(\mathbf{x}) u_{j}
$$

where the shape functions are defined as:

$$
\boldsymbol{\Phi}(\mathbf{x})=\left[\emptyset_{1}(\mathbf{x}) \ldots \emptyset_{n}(\mathbf{x})\right]=\mathbf{P}^{T}\left(\mathbf{P}^{T} \mathbf{W P}\right)^{-1} \mathbf{P}^{T} \mathbf{W}
$$

It should be noted that the approximation in equation (12) and the shape functions are not polynomials even if the bases $\mathbf{p}(\mathbf{x})$ are polynomials.

The weight function plays an important role in the formulation of the MLS approximation: it provides weightings for the residuals at different nodes within the (compact) support domain and it ensures that nodes enter and leave the influence domain smoothly so that the shape functions satisfy the compatibility condition and the approximation is globally continuous.

As shown by (13) the shape functions can be constructed only if the moment matrix (10) is non-singular. The necessary conditions for the moment matrix to be non- 
singular depend on the bases used. For linear bases, as in (3), the moment matrix is nonsingular as long as in the support domain there are at least three non-collinear nodes (in 2D). For the quadratic bases in (4), the conditions for obtaining a non-singular moment matrix are more complex. At least six nodes are needed in the support domain (in 2D), but even if more nodes are included, some nodal distributions can still lead to singular moment matrices (for example nodes distributed on two parallel lines).

To avoid the nodal configurations which lead to a singular moment matrix, the usual solution is to enlarge the support domains in order to include more nodes. The proposed MMLS method will avoid singular moment matrices for higher order polynomial bases by adding additional constraints to the error functional, as shown in the following section. 


\subsection{A modified MLS approximation method for second order polynomial bases}

\subsubsection{Shape functions derivation}

For simplicity, the following derivation will be made for bivariate data, but can be easily extended to higher dimensions. A singular moment matrix basically means that equation (9) used to compute the coefficients $\mathbf{a}(\mathbf{x})$ has multiple solutions, and therefore the functional (1) does not include sufficient constraints to guarantee a unique solution for the given node distribution. Based on this observation we propose to add additional constraints to the functional (1) as follows:

$$
\bar{J}(\mathbf{x})=\sum_{j=1}^{n}\left[\left(u^{h}\left(\mathbf{x}_{j}\right)-u_{j}\right)^{2} w\left(\left\|\mathbf{x}-\mathbf{x}_{j}\right\|\right)\right]+\mu_{x^{2}} a_{x^{2}}^{2}+\mu_{x y} a_{x y}^{2}+\mu_{y^{2}} a_{y^{2}}^{2}
$$

where

$$
\boldsymbol{\mu}=\left[\begin{array}{lll}
\mu_{x^{2}} & \mu_{x y} & \mu_{y^{2}}
\end{array}\right]
$$

is a vector of positive weights for the additional constraints.

The choice of the additional constraints ensures that, when the classical MLS moment matrix is singular (multiple solutions), we favor the solution having the coefficients for the higher order monomials in the bases equal to zero. This has a similar effect to the procedure of bases terms elimination used in the MTA [2] - it ensures that a solution with zero coefficients for some higher order monomials is selected when the standard MLS minimization procedure has multiple solutions. By choosing the weights for the additional constraints as small positive numbers we can ensure that the classical MLS solution is little changed when the moment matrix is not singular.

Therefore, the new functional can be rewritten in matrix form as

$$
\bar{J}=(\mathbf{P a}-\mathbf{u})^{T} \mathbf{W}(\mathbf{P a}-\mathbf{u})+\mathbf{a}^{T} \mathbf{H a}
$$

where $\mathbf{H}$ is a matrix with all elements equal to zero except the last 3 diagonal entries, equal to $\boldsymbol{\mu}$ : 


$$
\mathbf{H}=\left[\begin{array}{cc}
\mathbf{0}_{33} & \mathbf{0}_{33} \\
\mathbf{0}_{33} & \operatorname{diag}(\boldsymbol{\mu})
\end{array}\right]
$$

Following the same solution method as before, the minimization of the modified functional gives:

$$
\frac{\partial \overline{\mathbf{J}}}{\partial \mathbf{a}}=\left[\mathbf{P}^{T} \mathbf{W} \mathbf{P}+\mathbf{H}\right] \mathbf{a}(\mathbf{x})-\mathbf{P}^{T} \mathbf{W u}=0
$$

Therefore, as long as the modified moment matrix

$$
\overline{\mathbf{M}}=\mathbf{P}^{T} \mathbf{W P}+\mathbf{H}=\mathbf{M}+\mathbf{H}
$$

is non-singular, the new coefficients can be computed as:

$$
\mathbf{a}(\mathbf{x})=\left(\mathbf{P}^{T} \mathbf{W} \mathbf{P}+\mathbf{H}\right)^{-1} \mathbf{P}^{T} \mathbf{W u}
$$

The modified approximant becomes

$$
\bar{u}^{h}(\mathbf{x})=\mathbf{P}^{T}\left(\mathbf{P}^{T} \mathbf{W} \mathbf{P}+\mathbf{H}\right)^{-1} \mathbf{P}^{T} \mathbf{W} \mathbf{u}=\sum_{j=1}^{n} \bar{\emptyset}_{j}(\mathbf{x}) u_{j}
$$

with the new shape functions:

$$
\overline{\boldsymbol{\Phi}}(\mathbf{x})=\left[\begin{array}{lll}
\bar{\emptyset}_{1}(\mathbf{x}) & \ldots & \bar{\emptyset}_{n}(\mathbf{x})
\end{array}\right]=\mathbf{P}^{T}\left(\mathbf{P}^{T} \mathbf{W} \mathbf{P}+\mathbf{H}\right)^{-1} \mathbf{P}^{T} \mathbf{W}
$$

\subsubsection{Comparison with Tikhonov-Miller regularization}

The Tikhonov-Miller regularization [12-14] can be extended to quadratic basis, resulting in the same relations as above; the only difference would be the Tikhonov matrix $\mathbf{H}$, chosen as a multiple of the identity matrix $\mathbf{H}^{*}=\mu \mathbf{I}$. The matrix $\mathbf{H}$ derived using the above procedure confers the obtained shape functions special properties which make them appropriate for use in meshless methods, such as being a partition of unity and having the linear field reproduction property [2]. We will not derive these properties in this paper; only the properties important for scattered data interpolation will be presented in the next Section. 


\subsection{Properties of the Modified MLS approximation}

In this section we demonstrate some useful and important properties of the proposed modified MLS.

\subsubsection{Acceptable nodal distribution}

We will consider a nodal distribution as acceptable only if the moment matrix is nonsingular, allowing the computation of shape functions at any point in the domain.

Lemma 1: A nodal distribution which is acceptable for the classical MLS method with linear bases is also acceptable for the new modified MLS method with quadratic bases.

\section{Proof:}

The moment matrix given by (19) can be rewritten as:

$$
\overline{\mathbf{M}}=\overline{\mathbf{P}}^{T} \overline{\mathbf{W}} \overline{\mathbf{P}}
$$

with

$$
\mathbf{P}=\left[\begin{array}{cccccc}
1 & x_{1} & y_{1} & y_{1}{ }^{2} & x_{1} y_{1} & y_{1}{ }^{2} \\
\vdots & \vdots & \vdots & \vdots & \vdots & \vdots \\
1 & x_{n} & y_{n} & x_{n}{ }^{2} & x_{n} y_{n} & y_{n}{ }^{2} \\
0 & 0 & 0 & 1 & 0 & 0 \\
0 & 0 & 0 & 0 & 1 & 0 \\
0 & 0 & 0 & 0 & 0 & 1
\end{array}\right]
$$

and

$$
\overline{\mathbf{W}}=\left[\begin{array}{cc}
\mathbf{W} & \mathbf{0}_{n 3} \\
\mathbf{0}_{3 n} & \operatorname{diag}(\boldsymbol{\mu})
\end{array}\right]
$$

Equation (23) can be further transformed into:

$$
\overline{\mathbf{M}}=\overline{\mathbf{P}}^{T} \mathbf{Y}^{T} \mathbf{Y} \overline{\mathbf{P}}=\mathbf{R}^{T} \mathbf{R}
$$

where

$$
\mathbf{Y}=\operatorname{sqrt}(\mathbf{W}), \quad \mathbf{R}=\mathbf{Y} \overline{\mathbf{P}}
$$

Based on the matrix rank properties, because $\mathbf{Y}$ is a diagonal matrix with non-zero (positive) diagonal elements (based on (25), (27), the positive $\mathbf{W}$ and $\boldsymbol{\mu}$ ):

$$
\operatorname{rank}(\mathbf{R})=\operatorname{rank}(\overline{\mathbf{P}})
$$

From (26): 


$$
\operatorname{rank}(\overline{\mathbf{M}})=\operatorname{rank}\left(\mathbf{R}^{T} \mathbf{R}\right)=\operatorname{rank}(\mathbf{R})
$$

and therefore, by combining (28) and (29):

$$
\operatorname{rank}(\overline{\mathbf{M}})=\operatorname{rank}(\overline{\mathbf{P}})
$$

Equation (30) shows that in order for the modified moment matrix to be nonsingular, matrix $\overline{\mathbf{P}}$ needs to have full rank (six in our case). Based on its definition (24), matrix $\overline{\mathbf{P}}$ has full rank only if matrix

$$
\mathbf{P}_{1}=\left[\begin{array}{ccc}
1 & x_{1} & y_{1} \\
\vdots & \vdots & \vdots \\
1 & x_{n} & y_{n}
\end{array}\right]
$$

has full rank. This condition is the same as the condition needed for the classical MLS method with linear bases to have a non-singular moment matrix - the support domain needs to contain at least three non-collinear nodes.

This demonstration can be easily extended to $3 \mathrm{D}$, where the moment matrix is non-singular if the support domain contains at least four non-coplanar nodes.

These restrictions on nodal distribution are a lot less severe than the restrictions for the classical MLS method with quadratic bases. The shape functions can be computed based on a reduced number of nodes, allowing smaller support domains and increased computational efficiency of the method.

\subsubsection{Continuity of approximation}

One of the major advantages of MLS approximation is its compatibility, which means the approximation field function is continuous and smooth in the entire problem domain.

Lemma 2: Let $w^{j}(\mathbf{x})=w\left(\left\|\mathbf{x}-\mathbf{x}_{j}\right\|\right) \in C^{l}(\Omega)$ (derivatives up to order $l$ are continuous). If $\boldsymbol{\mu}$ is a constant vector and the moment matrix $\overline{\mathbf{M}}$ is invertible at every point of $\Omega$, then $\bar{u}^{h}(\mathbf{x}) \in C^{l}(\Omega)$

\section{Proof:}

From Equation (21), the approximation $\bar{u}^{h}(\mathbf{x})$ on the whole problem domain is a span of all shape functions. Therefore, like for traditional MLS, the smoothness (or the 
order of continuity) of the approximation equals the smoothness of the shape functions, which is determined by the functions with the minimum order of continuity in (22). Following the assumption that the moment matrix $\mathbf{P}^{T} \mathbf{W P}+\mathbf{H}$ is non-singular and since the monomials in the bases have $C^{\infty}$ continuity, the smoothness of the shape functions is determined by the weight function and the vector $\boldsymbol{\mu}$ (which defines matrix $\mathbf{H}$ ). As a constant, $\boldsymbol{\mu}$ has $C^{\infty}$ continuity, therefore the smoothness of the approximation function is solely determined by the smoothness of the weight functions, similar to the classical MLS approximation. 


\section{NUMERICAL EXPERIMENTS}

The modified MLS (MMLS) method has been implemented in Matlab for 1D and 2D. A quartic weight function with a circular domain is used:

$$
w(s)= \begin{cases}1-6 s^{2}+8 s^{3}-3 s^{4} & , s \leq 1 \\ 0 & , s>1\end{cases}
$$

where $s$ is the normalized distance

$$
s_{j}=\frac{\left\|\mathbf{x}-\boldsymbol{x}_{j}\right\|}{R_{j}}
$$

and $R_{j}$ is the radius of influence domain of node $\mathbf{x}_{j}$ [2]. For simplicity, in our implementations we use the same weights for all the additional constraints

$$
\mu_{x^{2}}=\mu_{x y}=\mu_{y^{2}}=\mu
$$

and a constant radius of influence for all nodes $R_{j}=R$ (as we use nodal distributions with an almost constant density of nodes within the domain).

\subsection{MLS and modified MLS (MMLS) shape functions in 1D}

A comparison between the MLS, Tikhonov-Miller regularization and the MMLS shape functions is presented in Fig. 1 for different values of $\mu$ and $R$. For the radius of influence, the larger value $(R=2.5)$ was chosen such that more than three nodes are in the support domain of any point in the interval, while the lower value $(R=1.3)$ only ensures that two nodes are in the support domain of any point in the interval. For small radius of influence $R$ the classical MLS with quadratic base functions has singular moment matrix for parts of the domain, while the modified MLS does not have such a problem. The value of the weight parameter $\mu$ influences the shape functions: the smaller the value, the closer the MMLS shape functions are to those of the classical MLS with quadratic base functions (when these exist). The Tikhonov-Miller regularization shape functions do not form a partition of unity. 


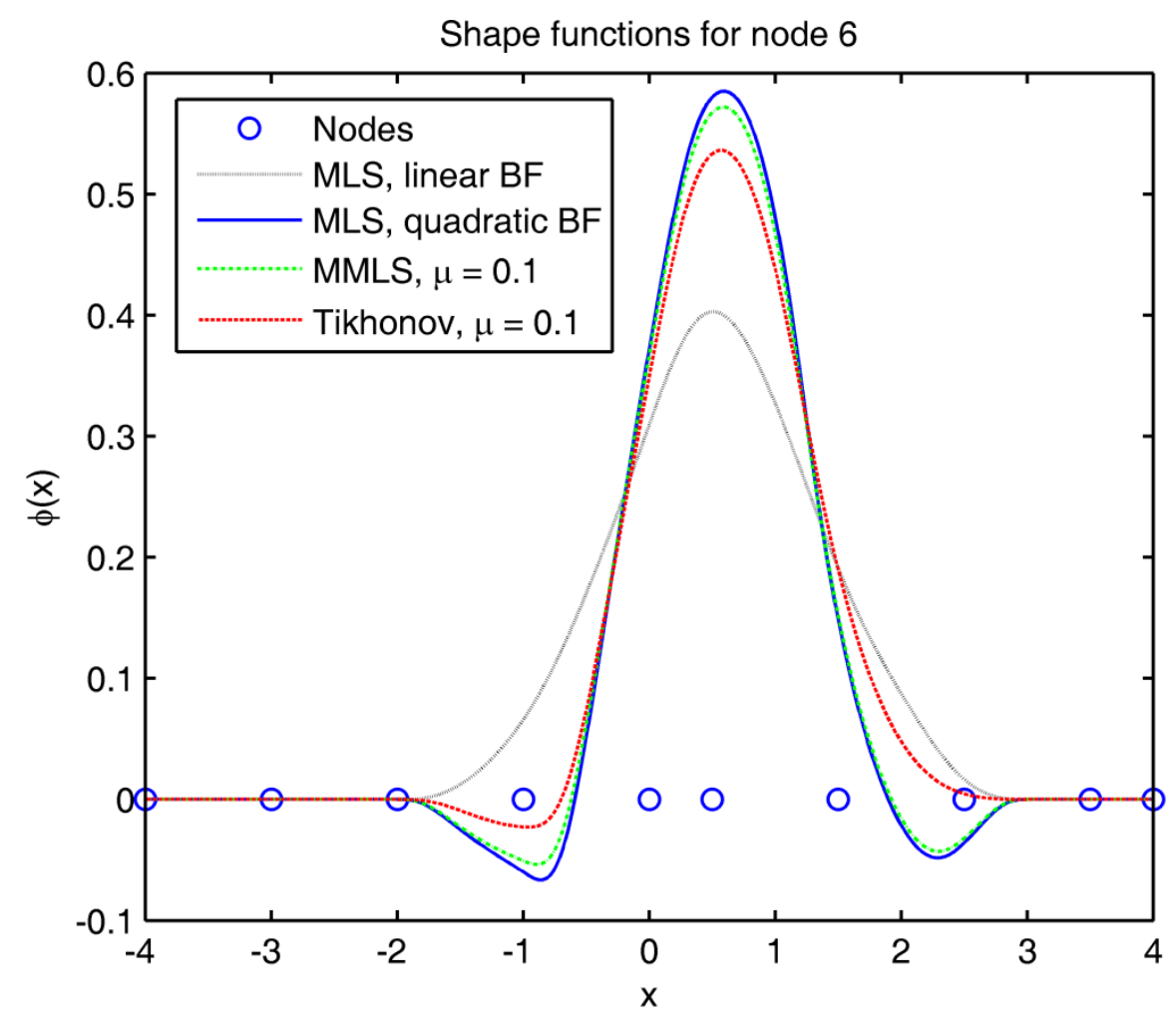

a)

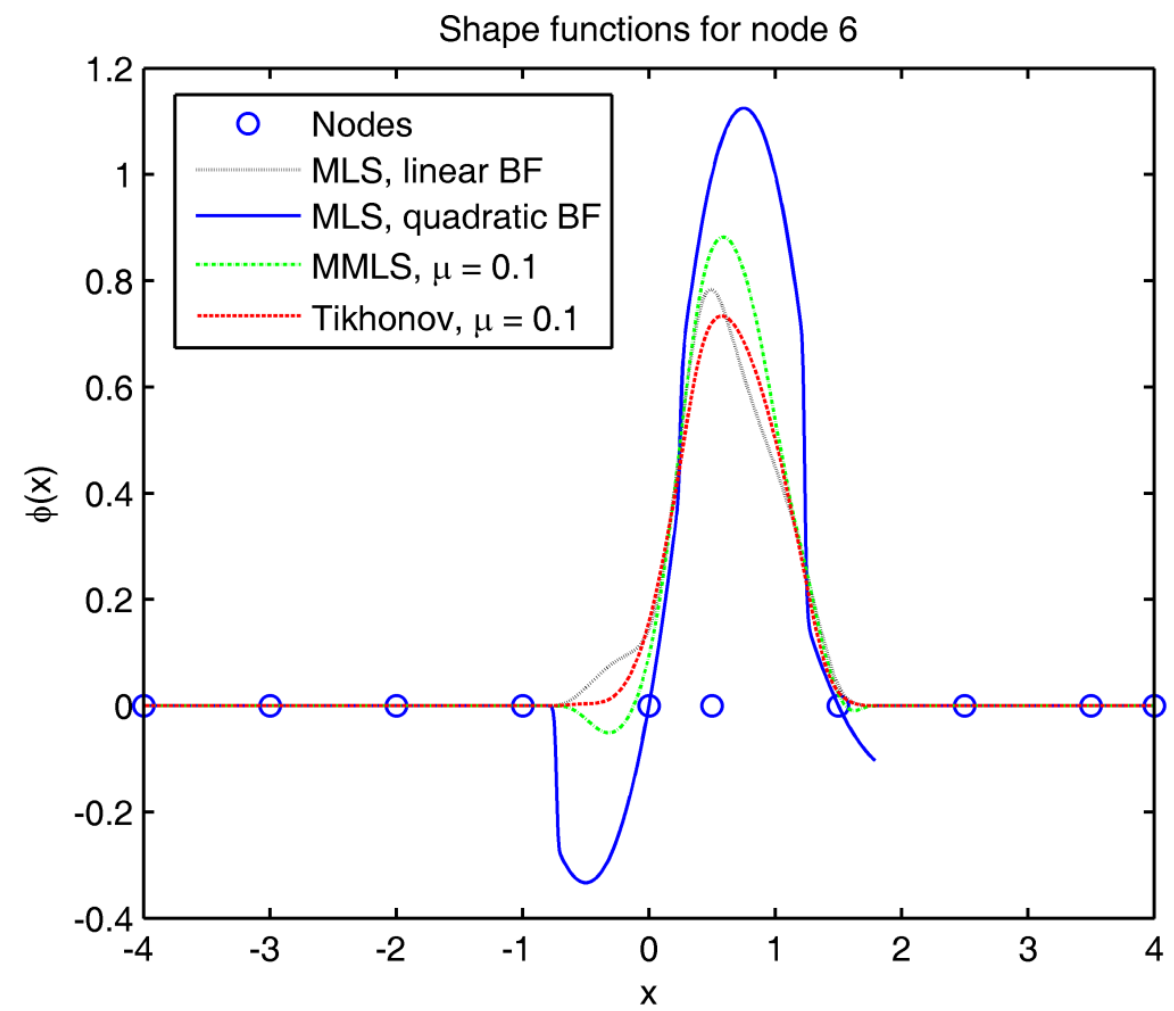

b)

Fig. 1 MLS, Tikhonov-Miller regularization and MMLS shape functions comparison for node 6. a) $R=2.5$. b) $R=1.3$; the classical MLS with quadratic base functions (BF) has a singular moment matrix for part of the domain 


\subsection{Approximation accuracy in $1 D$}

Using the same nodal distribution as in the above example, we approximated a non-polynomial function, $u(x)=\sin (x)$, using MLS, Tikhonov-Miller regularization and the MMLS, for different values of $\mu$ and $R$. The approximation accuracy was determined using the root mean square error

$$
R M S E=\sqrt{\frac{\sum_{i=1}^{N}\left(u\left(\mathbf{x}_{i}\right)-u^{h}\left(\mathbf{x}_{i}\right)\right)^{2}}{N}}
$$

computed using $\mathrm{N}=801$ points equally distributed in the interval $[-4,4]$. The results are presented in Fig. 2 and Table 1.

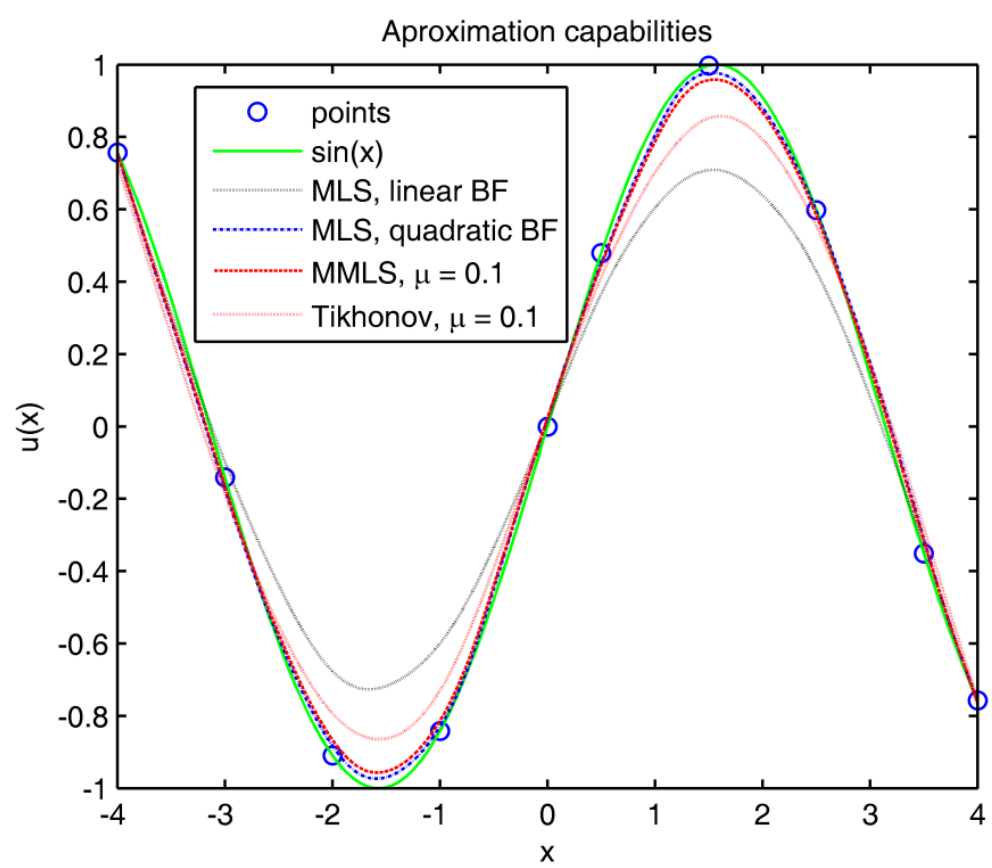

Fig. 2 Approximation capability for MLS, Tikhonov-Miller regularization and MMLS, $R=2.5$

Table 1. Root mean square error in approximating $u(x)=\sin (x)$ using the points presented in Fig. 2 .

\begin{tabular}{|c|c|c|}
\hline \multirow{2}{*}{ Approximation method } & \multicolumn{2}{|c|}{ Radius of nodal influence domain, $\boldsymbol{R}$} \\
\cline { 2 - 3 } & $\mathbf{2 . 5}$ & $\mathbf{1 . 3}$ \\
\hline MLS, linear BF & 0.1765 & 0.0597 \\
\hline MLS, quadratic BF & 0.0297 & Singular M \\
\hline Tikhonov-Miller, $\mu=0.1$ & 0.0922 & 0.0934 \\
\hline Tikhonov-Miller, $\mu=0.01$ & 0.0488 & 0.0603 \\
\hline MMLS, $\mu=0.1$ & 0.0355 & 0.0559 \\
\hline MMLS, $\mu=0.01$ & 0.0301 & 0.0501 \\
\hline
\end{tabular}


The results show that the approximation accuracy of the MMLS is better than that of the classical MLS with linear base functions, approaching the accuracy of classical MLS with quadratic base functions as the value of parameter $\mu$ decreases. Fig. 2 clearly shows the advantage of using higher degree base functions in terms of approximation accuracy. The MMLS also leads to better approximation than Tikhonov-Miller regularization for the same values of the parameter $\mu$.

\subsection{Approximation accuracy in $2 D$}

The following function was used for testing the approximation accuracy in 2D:

$$
u(x, y)=\left(x^{2}-y^{2}\right) e^{-x^{2}-y^{2}}
$$

using MLS and the MMLS, for different values of $\mu$ and $R$. The chosen function combines rapid transitions between peaks and dips with almost flat regions over the domain. Both regular and irregular node distributions were used, consisting of 324 nodes, as shown in Fig. 3. The RMSE was computed using a regular distribution of $\mathrm{N}=81 * 81$ points. The results, presented in Table 2, support the conclusions drawn from the univariate results.

Table 2. Root mean square error in approximating $u(x, y)=\left(x^{2}-y^{2}\right) e^{-x^{2}-y^{2}}$ using the nodes presented in Fig. 3.

\begin{tabular}{|c|c|c|c|c|}
\hline \multirow{2}{*}{$\begin{array}{c}\text { Approximation } \\
\text { method }\end{array}$} & \multicolumn{3}{|c|}{ Regular node distribution } & \multicolumn{2}{c|}{ Irregular node distribution } \\
\cline { 2 - 5 } & \multicolumn{3}{|c|}{ Radius of nodal influence domain, $\boldsymbol{R}$} \\
\cline { 2 - 5 } & $\mathbf{1 . 5}$ & $\mathbf{0 . 8}$ & $\mathbf{1 . 5}$ & $\mathbf{0 . 8}$ \\
\hline MLS, linear BF & 0.0366 & 0.0136 & 0.0372 & 0.0168 \\
\hline MLS, quadratic BF & 0.0107 & Singular M & 0.0134 & Singular M \\
\hline MMLS, $\mu=0.1$ & 0.0158 & 0.0127 & 0.0185 & 0.0162 \\
\hline MMLS, $\mu=0.001$ & 0.0108 & 0.0058 & 0.0135 & 0.0091 \\
\hline MMLS, $\mu=0.0001$ & 0.0107 & 0.0053 & 0.0134 & 0.0062 \\
\hline
\end{tabular}




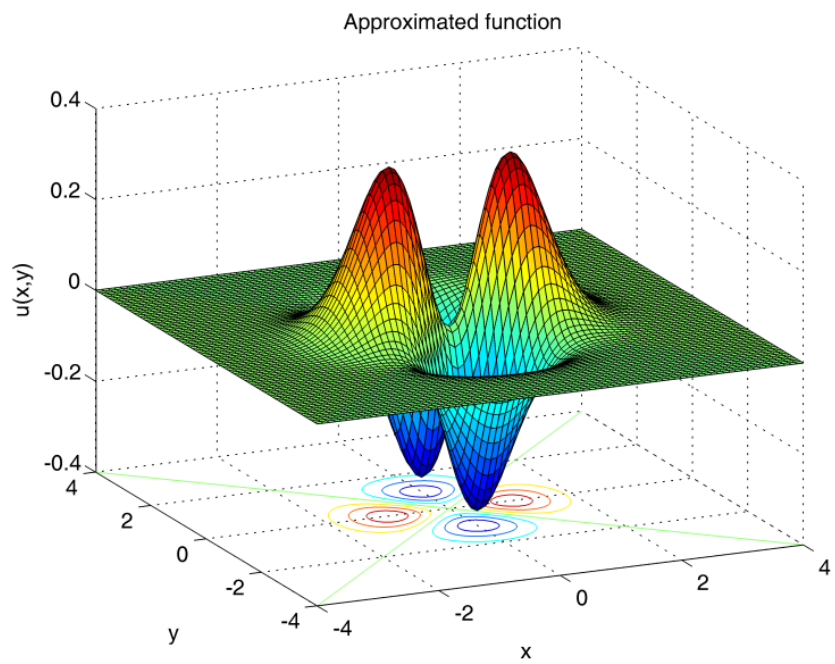

a)

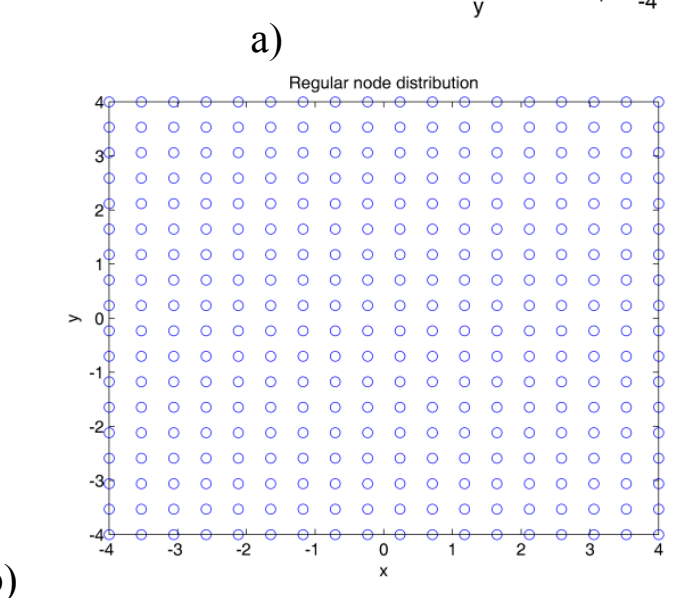

b)

Fig. 3 a) Approximated function - eq. (36). b) Regular node distribution. c) Irregular node distribution. The obtained approximation surfaces are visually identical to the approximated function.

\subsection{Accuracy evaluation of a meshless method based on MMLS}

We used the proposed MMLS and the classical MLS to implement a meshless method using a Total Lagrangian Explicit Dynamics framework [11]. We performed a simulation of a soft tissue extension using a 2D geometry $(10 \mathrm{~cm} \times 4 \mathrm{~cm})$. The problem domain was discretised using 57 nodes. To ensure integration accuracy, a dense regular background grid was used consisting of 4000 integration cells with one integration point per cell. For each node, the radius of the influence domain was constant $(R=1.4)$. The nodes on the right boundary were displaced by $3 \mathrm{~cm}$ while the left boundary nodes were 
fixed. The steady state solution was computed using adaptive dynamic relaxation [17, 18], with the stable time step estimated as described in [19].

Following $[17,20]$ a hyper-elastic Neo-Hookean material model was chosen to capture the behaviour of soft tissues undergoing large deformation. A similar finite element analysis was performed in the commercial finite element software Abaqus, with identical constitutive material law, loading and boundary conditions; the steady state solution was obtained using the static solver with the default configuration. The difference in computed displacement fields between the results obtained using the MLS and MMLS meshless methods and the reference results obtained using Abaqus are presented in Fig. 4 and Table 3.

a)

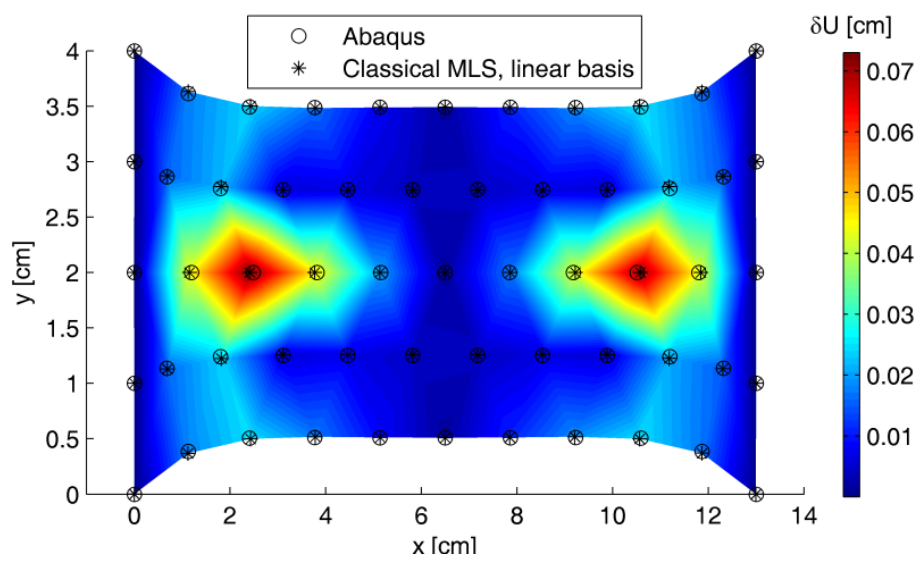

b)

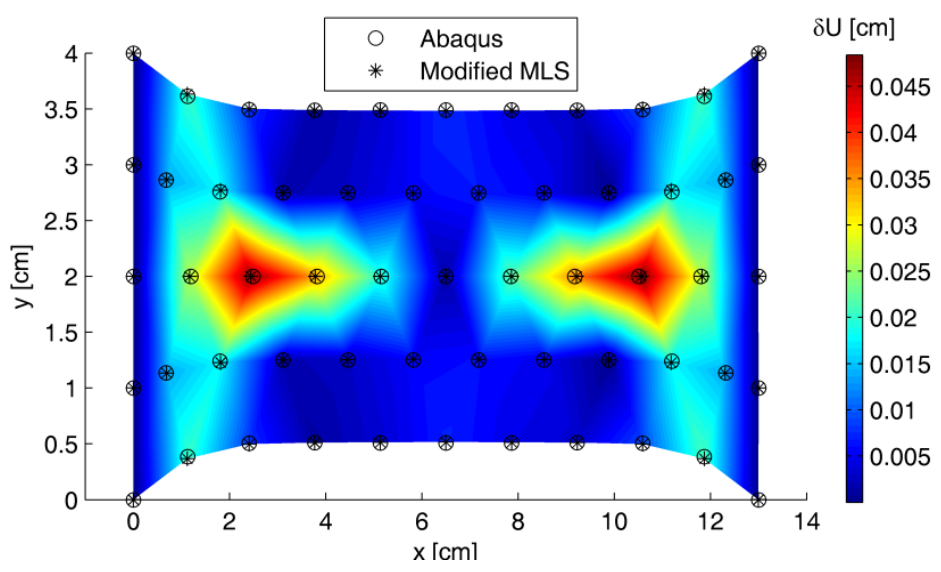

Fig. 4 Differences in the computed deformation field a) between classic MLS (linear basis) and Abaqus; b) between MMLS and Abaqus. 
Table 3. Difference in displacement fields between the meshless methods and Abaqus results.

\begin{tabular}{|l|c|c|}
\hline Approximation method & $\begin{array}{c}\text { Average } \\
\text { difference }(\mathbf{m m})\end{array}$ & $\begin{array}{c}\text { Maximum difference } \\
\text { (mm) }\end{array}$ \\
\hline Classical MLS & 0.15 & 0.73 \\
\hline MMLS $\left(\mu=10^{-10}\right)$ & 0.1 & 0.48 \\
\hline
\end{tabular}

For the given nodal influence domain radius, the classical MLS with quadratic basis failed due to the singularity of moment matrix. The results show that the maximum and average difference in displacements between MMLS and Abaqus are lower as compared to those between classic MLS using linear basis and Abaqus. 


\section{DISCUSSION AND CONCLUSIONS}

The modified Moving Least Squares (MMLS) presented in this paper is based on the error functional used in the derivation of the classical MLS approximation augmented with additional terms based on the coefficients of the polynomial base functions. This allows quadratic polynomial base functions to be used with the same size of the support domain as linear base functions, resulting in better approximation capability while maintaining the continuity and smoothness of the approximation.

The numerical examples show that the approximation accuracy of the MMLS is better than that of the classical MLS with linear base functions, approaching the accuracy of classical MLS with quadratic base functions as the value of parameter $\mu$ (the weights for the additional constraints) decreases. An important benefit of the proposed method is the ability to provide an approximation for cases when classical MLS with quadratic base functions fails due to a singular moment matrix.

The proposed method has been presented for bivariate functions and quadratic base functions for simplicity, but can be extended to 3D and higher order polynomial bases.

The proposed MMLS method is similar to Tikhonov-Miller regularization; compared to Tikhonov-Miller regularization, the MMLS has better approximation properties, the resulting shape functions form a partition of unity and have the linear field reproduction property, therefore being appropriate for use in meshless methods. We intend to use the presented MMLS approximation in the development of a more robust Element Free Garlekin meshless method [5, 11] for surgical simulations. The initial results show that the MMLS approximation leads to better solution accuracy as compared to the classical MLS approximation.

Acknowledgements: The financial support of the Australian Research Council (Discovery Grant No. DP120100402) and the National Health and Medical Research Council (Grants No. APP1063986 and APP1083572) is gratefully acknowledged. 


\section{BIBLIOGRAPHY}

[1] S. Li, W.K. Liu, Meshfree Particle Methods, Springer-Verlag, Berlin, 2004.

[2] G.R. Liu, Mesh Free Methods: Moving Beyond the Finite Element Method, CRC Press, Boca Raton, 2003.

[3] X. Jin, G.R. Joldes, K. Miller, K.H. Yang, A. Wittek, Meshless algorithm for soft tissue cutting in surgical simulation, Comput. Methods Biomech. Biomed. Engin., 17 (2014) 800-811.

[4] G. Zhang, A. Wittek, G.R. Joldes, X. Jin, K. Miller, A three-dimensional nonlinear meshfree algorithm for simulating mechanical responses of soft tissue, Eng. Anal. Bound. Elem., 42 (2014) 60-66.

[5] K. Miller, A. Horton, G.R. Joldes, A. Wittek, Beyond finite elements: a comprehensive, patient-specific neurosurgical simulation utilizing a meshless method, J. Biomech., 45 (2012) 2698-2701.

[6] P. Lancaster, K. Salkauskas, Surfaces generated by moving least squares methods, Math. Comput., 37 (1981).

[7] M. Alexa, J. Behr, D. Cohen-Or, S. Fleishman, D. Levin, C.T. Silva, Computing and rendering point set surfaces, IEEE Transactions on Visualization and Computer Graphics, 9 (2003) 3-15.

[8] C. Shen, J.F. O'Brien, J.R. Shewchuk, Interpolating and approximating implicit surfaces from polygon soup, ACM Trans. Graph., 23 (2004) 896-904.

[9] M. Muller, R. Keiser, A. Nealen, M. Pauly, M. Gross, M. Alexa, Point based animation of elastic, plastic and melting objects, Proceedings of the 2004 ACM SIGGRAPH/Eurographics symposium on Computer animation, Eurographics Association, Grenoble, France, 2004, pp. 141-151.

[10] T. Belytschko, Y. Krongauz, D. Organ, M. Fleming, P. Krysl, Meshless methods: An overview and recent developments, Comput. Methods Appl. Mech. Eng., 139 (1996) 3-47.

[11] A. Horton, A. Wittek, G.R. Joldes, K. Miller, A Meshless Total Lagrangian Explicit Dynamics Algorithm for Surgical Simulation, Int. J. Numer. Method. Biomed. Eng., 26 (2010) 977-998.

[12] W.H. Press, S.A. Teukolsky, W.T. Vetterling, B.P. Flannery, Section 19.5. Linear Regularization Methods, Numerical Recipes: The Art of Scientific Computing, Cambridge University Press, New York, 2007. 
[13] A.N. Tikhonov, V.Y. Arsenin, Solution of Ill-posed Problems, Winston \& Sons, Washington, 1977.

[14] K. Miller, Least Squares Methods for Ill-Posed Problems with a Prescribed Bound, SIAM Journal on Mathematical Analysis, 1 (1970) 52-74.

[15] R. Tibshirani, Regression Shrinkage and Selection via the Lasso, Journal of the Royal Statistical Society. Series B (Methodological), 58 (1996) 267-288.

[16] H. Zou, T. Hastie, Regularization and variable selection via the elastic net, Journal of the Royal Statistical Society: Series B (Statistical Methodology), 67 (2005) 301-320.

[17] G.R. Joldes, A. Wittek, K. Miller, Computation of intra-operative brain shift using dynamic relaxation, Computer Methods in Applied Mechanics and Engineering, 198 (2009) 3313-3320.

[18] G.R. Joldes, A. Wittek, K. Miller, An adaptive Dynamic Relaxation method for solving nonlinear finite element problems. Application to brain shift estimation., Int. J. Numer. Method. Biomed. Eng., 27 (2011) 173-185.

[19] G.R. Joldes, A. Wittek, K. Miller, Stable time step estimates for mesh-free particle methods, Int. J. Numer. Meth. Eng., 91 (2012) 450-456.

[20] G.R. Joldes, A. Wittek, K. Miller, Suite of finite element algorithms for accurate computation of soft tissue deformation for surgical simulation, Med. Image Anal., 13 (2009) 912-919. 